\title{
The TRIP database
}

\author{
Derek Richards \\ Centre for Evidence-based Dentistry, Oxford, UK
}

\author{
The TRIP (Turning Research into Practice) database has an apparently simple goal: \\ to allow health professionals to easily find the highest-quality material available on \\ the Web to help support evidence-based practice. \\ Evidence-Based Dentistry (2008) 9, 28-29. doi:10.1038/sj.ebd.6400572
}

The TRIP database (Figure 1) grew out of the work of its founders Jon Brassey and Dr Chris Price, trying to answer clinical questions using the principles of evidence-based medicine. As a wide range of websites were used to identify the best available evidence, each website used took at least $5 \mathrm{~min}$ to search, and there was a need to respond to the questions in a clinically relevant time, the value was recognised of having just one website to search. Consequently, the first, crude, web-based version of TRIP was launched in 1997. Since then, there have been a number of upgrades to the site and more recently the site has been externally evaluated by a team from the Centre for Evidence-based Medicine (www.cebm.net) led by Professor Paul Glasziou.
The TRIP database was a subscriptionbased service for 4 years, during which time a number of significant improvements were made but when use of the service also dropped off dramatically. Since September 2006, the database has been accessible to all.

\section{Database content}

Content on the database is identified in a variety of ways with regular systematic searches of the internet and regular collaboration with clinical answering services, such as the UK National Health Service clinical answering services (www.clinicalanswers.nhs.uk) and Attract (www.attract.wales.nhs.uk) to identify quality resources. TRIP also works with the Department of Family Medicine at Laval University, Quebec, Canada, to help identify and appraise sites in a more systematic man- ner. A directory of evaluated websites using a validated evaluation instrument is available at http://132.203.128.28/medecine/ repertoire/repertoire.asp.

The content of the TRIP database is separated into a number of categories (see Table 1), the divisions being based on Haynes' work on the ' $4 \mathrm{~S}$ ' approach to current best evidence. $^{1}$

\section{Searching TRIP}

The TRIP database is searched using a prominent search box that will be familiar to all those who regularly search internet sites. The usefulness of the site can be seen by the fact that an example-search I tried on fluoride varnish very rapidly identified the following:

- Systematic reviews 17

- Guidelines

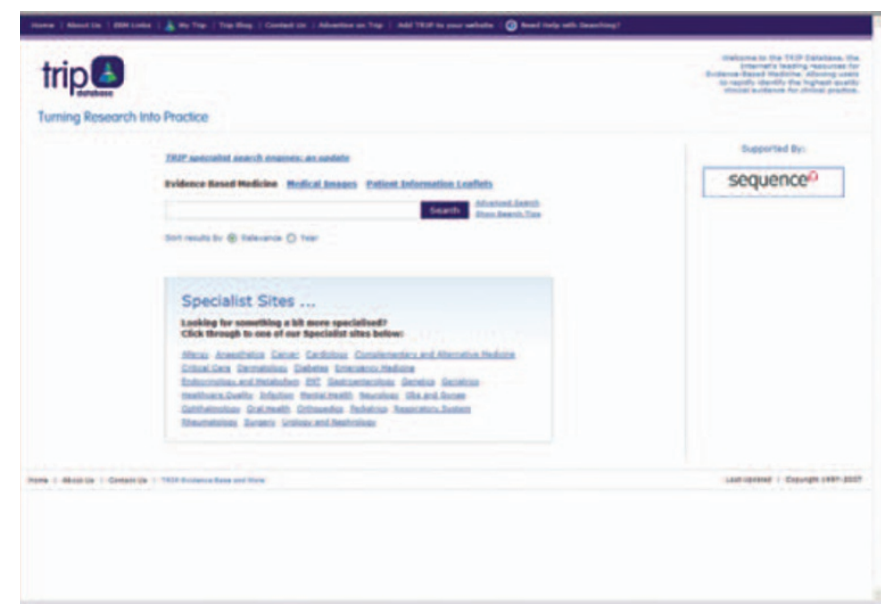

Figure 1. The TRIP database homepage

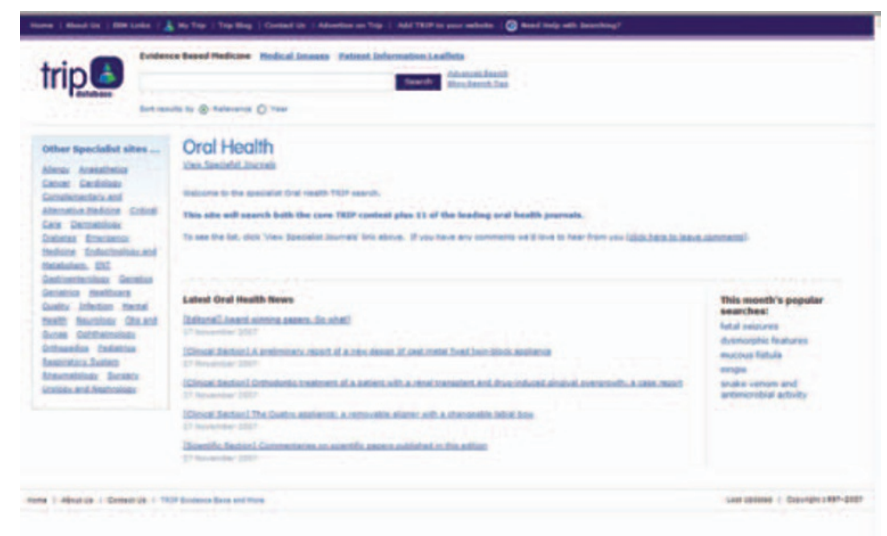


North America 4

Other 1

- Clinical questions 4

In addition to the following Medline articles:

- Therapy 85

- Diagnosis 2

- Systematic reviews 19

- Prognosis 5

- Aetiology 6

A similar search of PubMed for fluoride varnish produced 486 hits with 39 suggested to be reviews. In order to improve the PubMed search to identify the higher quality articles, a more detailed search with additional filters would be required. So for the busy clinician, higher quality information is identified more rapidly using TRIP.

A further refinement of the searching process on TRIP is the opportunity to search the specialist sites. Oral health is included as one of the specialist sites (Figure 2) and it searches both the core TRIP content and 11 leading oral health journals (see Table 2).

The TRIP database is produced by TRIP Database Limited, who have been involved for a number of years in healthrelated knowledge management. Their TRIP database is a very useful resource for the practitioner and one which should be included as one of your favourite sites or bookmarks.

1. Haynes RB. Of studies, syntheses, synopses, and

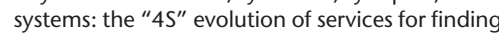
current best evidence. Evid Based Med 2001; $6: 36-38$.
Table 1. TRIP database categories

\begin{tabular}{l|l}
\hline Category & Description \\
\hline Evidence-based synopses & $\begin{array}{l}\text { Synopses of individual studies that have been critically } \\
\text { appraised. Includes evidence-based journal reviews, critically } \\
\text { appraised topics, clinical evidence }\end{array}$ \\
\hline Clinical answers & $\begin{array}{l}\text { A number of services exist to answer clinical questions. These } \\
\text { aim to match the best available evidence to the question }\end{array}$ \\
\hline Systematic reviews & Explicit, rigorous syntheses of primary research studies \\
\hline Guidelines & $\begin{array}{l}\text { Clinical guidelines gathered from collections from around the } \\
\text { globe separated according to country/ area of origin }\end{array}$ \\
\hline eTextbooks & $\begin{array}{l}\text { Online textbooks tend to be excellent sources of } \\
\text { background material }\end{array}$ \\
\hline Clinical calculators & Eg. odds ratio calculator, growth calculator, Glasgow coma scale \\
\hline Medline & $\begin{array}{l}\text { The database uses a special interface and validated search filters } \\
\text { to search PubMed and return highly-focused results }\end{array}$ \\
\hline TRIP, Turning Research into Practice. \\
\hline
\end{tabular}

\section{Table 2. Dental journals included in the oral health specialist search}

Journal title

Journal of Dental Research

British Dental Journal

Journal of the American Dental Association

Journal of Evidence Based Dental Practice

Evidence-based Dentistry

Journal of Clinical Periodontology

Oral Surgery Oral Medicine Oral Pathology Oral Radiology

Community Dentistry and Oral Epidemiology

International Journal of Paediatric Dentistry

Journal of Endodontics

Critical Reviews in Oral Biology and Medicine 